ORIGINAL ARTICLE

\title{
The neorule: a new instrument to measure linear growth in preterm infants
}

\author{
C J Lawn, R J Chavasse, K A Booth, M Angeles, F J Weir
}

Arch Dis Child Fetal Neonatal Ed 2004;89:F360-F363. doi: 10.1136/adc.2002.019448

See end of article for authors' affiliations

.....................

Correspondence to:

Dr Lawn, Trevor Mann

Baby Unit, Royal Sussex

County Hospital, Eastern

Road, Brighton BN2 5BE,

UK; cassie.lawn@bsuh.

nhs.uk

Accepted

15 September 2003
Objectives: To compare measurements of crown-heel length $(\mathrm{CHL})$ made with the neorule with $\mathrm{CHL}$ measurements made with a stadiometer in term infants. To examine safety and reproducibility of $\mathrm{CHL}$ measurements in infants $<32$ weeks gestational age (GA) using the neorule.

Methods: Three measurements of $\mathrm{CHL}$ were made by three teams during the first 2 days of life in healthy term infants. One team used the stadiometer and two the neorule. Two different teams made three measurements of $\mathrm{CHL}$ on four occasions at two week intervals in infants less than 32 weeks GA. Infants were continuously monitored, and any adverse event was recorded.

Results: Fifty term infants were studied, median (range) birth weight 3440 (2020-5010) g. The mean (SD, $95 \%$ confidence interval) difference between values obtained with the stadiometer and neorule was 0.08 $(6.22,-1.69$ to +1.85$) \mathrm{mm}$ and between the two neorule teams was $0.8(4.48,-0.47$ to +2.08$) \mathrm{mm}$. Twenty preterm infants were studied, GA median (range) $29\left(25^{+0}-31^{+6}\right)$ weeks, median (range) CHL 397 $(339-475) \mathrm{mm}$. There were no adverse events. The difference (SD, 95\%Cl) between teams in the mean $\mathrm{CHL}$ measurement was $0.18(4.79,-1.02$ to +1.38$) \mathrm{mm}$, with interobserver limit of agreement -9.2 to $+9.6 \mathrm{~mm}$ and coefficient of variation $1.2 \%$. There were no significant differences between measurements made by single observers; the $F$ ratio was 0.449 ( $d f=61, p=0.6$ ).

Conclusion: The neorule is a safe and accurate way to measure $\mathrm{CHL}$ in newborn infants.
A ccurate assessment of growth in the early neonatal period is vital. Weight and head circumference are the most the commonly used measures of growth in this group. Weight measurements are significantly affected by changes in hydration ${ }^{1}$ and do not discriminate between changes in the components that affect weight-that is, water, carbohydrate, fat, protein, and minerals. ${ }^{2}$ Although head circumference reflects brain growth," the effect of "head sparing" during periods of nutritional stress limits its usefulness as an overall indicator of nutritional adequacy. ${ }^{4}$

The best overall indicator of lean body mass and nutritional adequacy is linear growth. ${ }^{56}$ Measurement of linear growth in the sick preterm infant is rarely undertaken because measurement of crown-heel length (CHL) by stadiometry requires considerable handling of the infant and its removal from the incubator.

Other measurements of linear growth such as foot length, elbow-wrist length, ${ }^{8}$ or knee-heel length ${ }^{10-13}$ rely on the assumption that growth of a single extremity reflects whole body growth. Recent evidence suggests that knee-heel length is less reproducible than CHL especially in larger infants and is a poor predictor of CHL. ${ }^{14}$

The neorule is a novel device designed by RC. It comprises a stiff ruler with a fixed headboard and sliding footplate (fig l). It was designed to measure CHL in the incubator from the side without lifting the infant, minimising handling and the risk of displacement of endotracheal tube or intravascular catheters. The first study aims to compare the neorule with stadiometry in term infants, and the second study aims to test the safety and accuracy of the neorule in infants $<32$ weeks gestational age (GA).

\section{PATIENTS AND METHODS}

Term infant study

CHL was measured in healthy term infants born at Royal Women's Hospital, Melbourne, on one occasion during the first 2 days of life. Verbal consent was obtained from parents (after discussion with the chairperson of the local ethics committee who approved the study subject to verbal parental consent). Measurements were made by three teams of observers comprising medical and midwifery staff. Team 1 used a standard infant stadiometer, and teams 2 and 3 used the neorule. Each team made three measurements, a total of nine, over a period of no more than 15 minutes. The infants were naked during the measurements and allowed to flex between each measurement. The three teams measured the infants sequentially in a quasi-randomised order. Two people performed the measurement, and a third read and recorded the result. The same person positioned the head for all three teams. The positioning of the infant and technique for measurement CHL was according to the standard described below. ${ }^{14-16}$ The other teams waited outside of the room while measurements were being taken to ensure each team was blind to the others' results.

The mean (SD) CHL was calculated for each team. Interdevice comparisons were made by calculating the mean (SD, 95\% confidence interval (CI)) difference in CHL and $95 \%$ limits of agreement ${ }^{17}$ between team $\mathrm{l}$ using the stadiometer and team 2 using the neorule. The maximum absolute difference was also calculated. Interobserver comparisons were made in the same way between teams 2 and 3, which both used the neorule.

\section{Preterm infant study}

Infants $<32$ weeks GA admitted to one neonatal unit (Royal Sussex County Hospital, Brighton) were enrolled after informed written parental consent was obtained. Ethical approval was granted for the study from the local ethical advisory committee. CHL was measured using the neorule by two teams (A and B) of observers as soon as possible after birth and at two week intervals for six weeks (four measurement episodes). Each team measured CHL three times. The

Abbreviations: $\mathrm{CHL}$, crown-heel length; GA, gestational age 


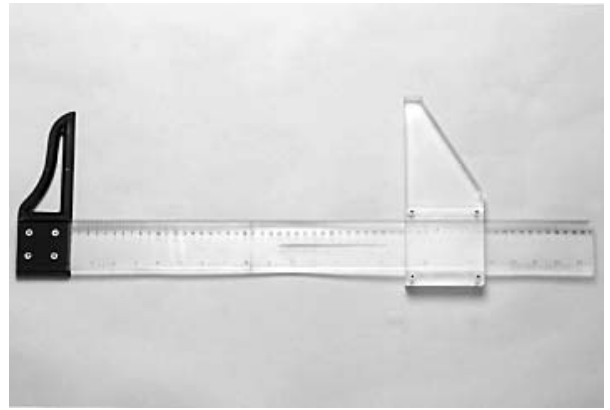

Figure 1 The neorule.

infant was allowed to flex between measurements. Each team consisted of two members of medical and nursing staff. CHL measurements were recorded by an observer who was not part of either team; individual teams waited outside the room while measurements were made by the other team to ensure each team was blind to the others' measurements. Infants were always measured in their incubator or cot while being nursed supine and were handled as little as possible. Swaddling and bedrolls were carefully removed and the baby was straightened to lie flat.

The positioning of the infant and technique for measuring CHL was according to the standard described below. ${ }^{14-16}$

Infants were continuously monitored for heart rate, respiratory rate, oxygen saturation, and temperature. Any significant clinical destabilisation marked by a fall in heart rate or oxygen saturation requiring intervention or adverse event such as displacement of intravascular catheter or endotracheal tube was recorded.

\section{Standard CHL measurement and positioning technique ${ }^{14-16}$}

Operator 1 holds the head gently with the eyes directly upwards with the lower margin of the orbit in the same vertical plane as the external auditory meatus and gently holds the headboard to the vertex. Operator 2 applies gentle traction to extend the legs without displacing the pelvis and then slides the footplate to meet the heel with just sufficient pressure to cause the skin to blanche; the CHL is then noted to the nearest $\mathrm{mm}$.

\section{Statistical analysis}

Interobserver reproducibility of the neorule

This was assessed by calculation of the differences in the maximum and the mean of each three CHL measurements made by each team on each occasion, and SD, maximum, 95\% CI, limit of agreement ${ }^{17}$ (mean (1.96 SD)), and coefficients of variation (SD/mean CHL) were calculated.

Intraobserver reproducibility of the neorule The difference between first and second and second and third measurements of CHL made by one team on each occasion were analysed, and a one way analysis of variance and coefficients of repeatability ${ }^{17}$ (2 SD differences) were calculated.

\section{RESULTS}

\section{Term infant study}

Fifty term infants (median (range) birth weight 3440 (2020$5010)$ g) were studied. The mean (SD) CHL by stadiometry was 502.7 (22.5) $\mathrm{mm}$. The mean (SD) CHLs measured by the neorule were 502.3 (22.1) $\mathrm{mm}$ and 502.1 (20.5) $\mathrm{mm}$ for teams 2 and 3 respectively.
The mean (SD, 95\% CI) difference between stadiometer and neorule (team 2) was $0.08(6.22,-1.69$ to +1.85$) \mathrm{mm}$ with $95 \%$ limits of agreement between -12.6 and $12.44 \mathrm{~mm}$. The maximum absolute difference was $17.3 \mathrm{~mm}$. The agreement between team 1 using the stadiometer and team 2 using the neorule is seen in the Bland-Altman plot of the difference in paired measurements plotted against the mean of those two measurements ${ }^{17}$ (fig 2).

The mean ( SD, 95\% CI) difference between neorule teams was $0.8(4.48,-0.47$ to +2.08$) \mathrm{mm}$ with $95 \%$ limits of agreement between -8.27 and $+9.88 \mathrm{~mm}$. The maximum absolute difference was $11.0 \mathrm{~mm}$. The agreement between teams 2 and 3 both using the neorule is seen in the BlandAltman plot of the difference in paired measurements plotted against the mean of those two measurements ${ }^{17}$ (fig 3).

\section{Preterm infant study}

Twenty babies were studied (11 boys, 9 girls). Median (range) GA was $29\left(25^{+0}-31^{+6}\right)$ weeks, and median (range) birth weight was 1302 (790-1720) g. The median (range) CHL was 397 (339-475) mm. At the first measurement episode, eight babies were intubated receiving intermittent positive pressure ventilation, five were receiving continuous positive airway pressure, and seven were self ventilating. No baby in this study received steroids during the trial period. Measurements were made on 61 occasions, 360 measurements in total. There were no adverse events.

The agreement between team 1 and team 2 is seen in the Bland-Altman plot of the difference in paired measurements plotted against the mean of those two measurements ${ }^{17}$ (fig 4).

The mean (SD, 95\% CI) difference between the maximum of three measurements taken by each team on each occasion was $0.2(4.91,-1.03$ to +1.43$) \mathrm{mm}$. The coefficient of variation was $1.2 \%$, and limit of agreement -9.4 to $+9.8 \mathrm{~mm}$. The greatest difference was $13 \mathrm{~mm}$.

The mean (SD, 95\% CI) difference between the means of three measurements taken by each team on each occasion was $0.18(4.79,-1.02$ to +1.38$) \mathrm{mm}$. The coefficient of variation was $1.2 \%$, and limit of agreement -9.2 to $+9.6 \mathrm{~mm}$. The greatest difference in means was $13.34 \mathrm{~mm}$.

Analysis of variance showed that there were no significant differences between three measurements taken by single observers; the $F$ ratio was $0.449(\mathrm{p}=0.6, \mathrm{df}=61)$.

Coefficients of repeatability were 4.5 and $4.2 \mathrm{~mm}$ - that is, $95 \%$ of second measurements made by a single observer were within $4.5 \mathrm{~mm}$ of the first measurement made by that

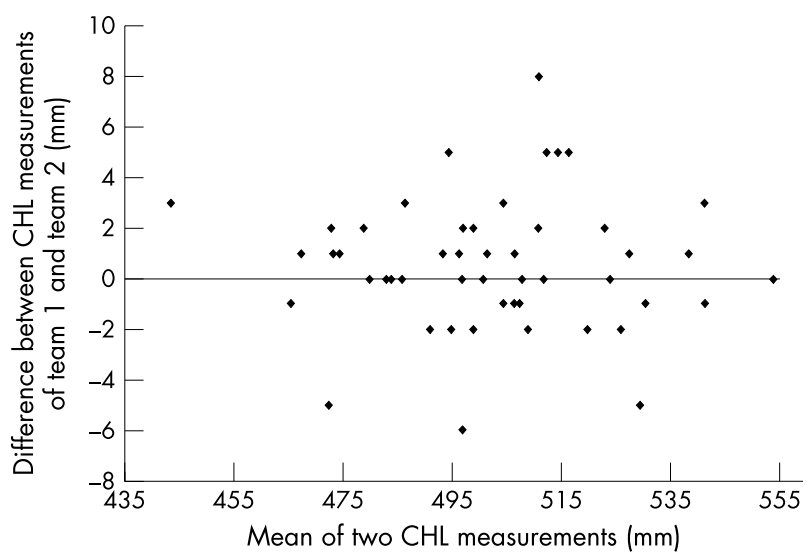

Figure 2 Term infant study. Bland-Altman plot of the difference between crown-heel length $(\mathrm{CHL})$ measurements made by team 1 (using the stadiometer) and team 2 (using the neorule) against the mean of those two CHL measurements. 


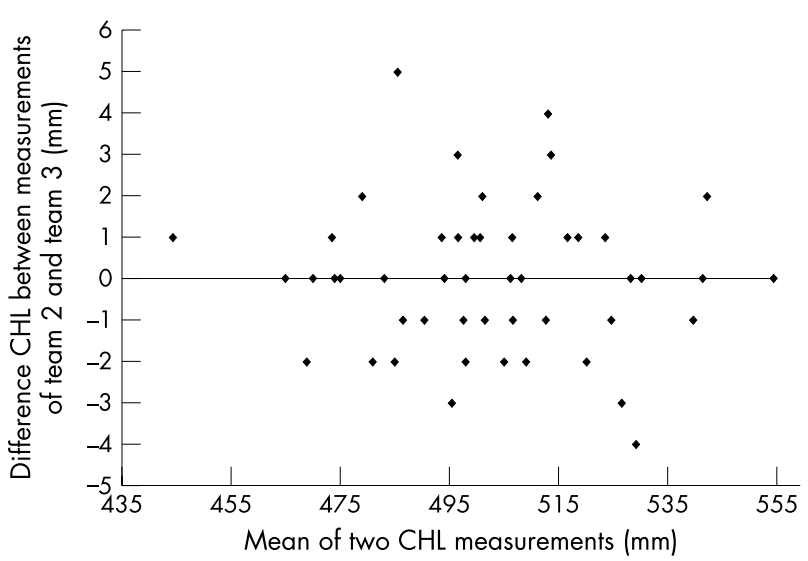

Figure 3 Term infant study. Bland-Altman plot of the differences in crown-heel length $(\mathrm{CHL})$ measurements made by team 2 and team 3 both using the neorule against the mean of those two $\mathrm{CHL}$ measurements.

observer and $95 \%$ of third measurements were within $4.2 \mathrm{~mm}$ of the second (fig 5).

\section{DISCUSSION}

CHL remains the best measurement of linear growth in infants as it is more reproducible than knee-heel length ${ }^{14}$ and does not rely on the assumption that growth of a single extremity reflects the growth of the whole body. Measurements of CHL in term infants rely on stadiometry. Stadiometry is rarely possible in all but the most stable infants under 32 weeks gestation, and therefore measurements of CHL in the preterm infant in neonatal intensive care are rarely undertaken. Extrauterine growth of very low birthweight infants is therefore incompletely monitored, and the impact of therapeutic manoeuvres-for example, respiratory support or steroids-and nutritional support is poorly understood.

Methods that do not require a specific device for measuring CHL in the preterm have been assessed. These include extending a tape measure along the infants body, placing the infant on a paper barrier and making a pencil mark at the head and foot and then measuring the distance between the marks and measuring crown-rump and then rump-heel length and adding these together. All these methods have poor reproducibility, with differences between observers that are less than $10 \mathrm{~mm}$ only occurring $60-62.5 \%$ of the time. ${ }^{18}$ The reproducibility of CHL measurements made with the neorule in infants $<32$ weeks GA compares favourably with paper tape methods, as $95 \%$ of duplicate maximum CHL measurements lie between -9.4 and $+9.8 \mathrm{~mm}$ using the neorule.

Devices specifically designed for use in the newborn are the prematometer, the neo-infantometer, and the neonatometer. The prematometer, designed for use in preterm infants, has been shown to be more reliable than measurements with a paper tape, ${ }^{19}$ and, although it can be used inside an incubator, it requires a clear space around the infant, which is rarely possible. The neo-infantometer also requires a clear space around the infant and has not been shown to have greater reproducibility than tape measure or paper barrier methods. ${ }^{20}$ The neonatometer allows highly reproducible measurements of CHL (coefficient of variation $=0.35 \%$ ), but requires removal of the infant from the incubator or $\cot ^{21}$

The neorule represents a considerable advance compared with these methods as it allows accurate, safe measurement of very preterm infants requiring intensive care without the

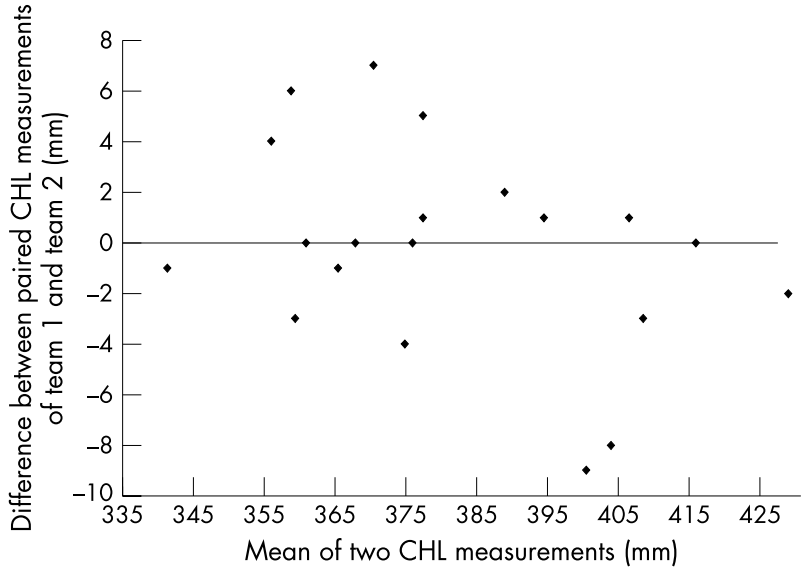

Figure 4 Preterm infant study. Bland-Altman plot of the difference between paired crown-heel length $(\mathrm{CHL})$ measurements $(\mathrm{mm})$ made by team 1 and team 2 against the mean of those two $\mathrm{CHL}$ measurements.

need to clear a space around them or remove them from the incubator.

We have also shown that the neorule allows CHL measurements in sick infants $<32$ weeks that are highly reproducible between observers. The maximum or mean of three measurements can be used with very similar accuracy.

Our study of term infants shows that the measurements of CHL using the neorule and stadiometer are comparable in term infants (the mean difference in CHL was $0.39 \mathrm{~mm}$ ) and that interobserver variability using the neorule in this age group was low (mean difference $0.8 \mathrm{~mm}$ ).

There were no significant differences between first, second, and third measurements made by single observers, so individual observers may take single measurements. However, in a clinical context, measurements need to be reproducible between observers, and three measurements of CHL are recommended. Plans to manufacture the neorule are under discussion, but it is not at present commercially available.

\section{- Difference between first and second $\mathrm{CHL}$ measurement $(\mathrm{mm})$ \\ - Difference between second and third $\mathrm{CHL}$ measurement $(\mathrm{mm})$}

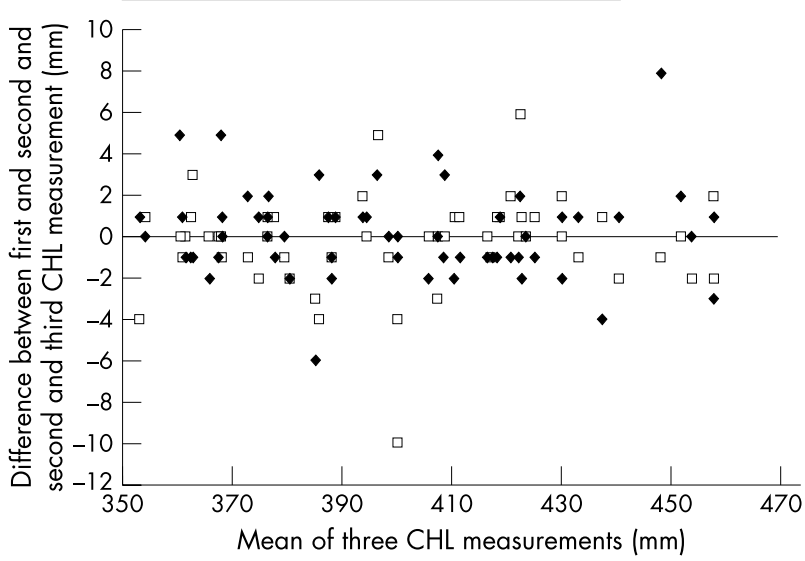

Figure 5 Preterm infant study. Bland-Altman plot of the differences between the first and second and second and third crown-heel length $(\mathrm{CHL})$ measurements by single observers against the mean of those three measurements of $\mathrm{CHL}$. 


\section{CONCLUSIONS}

The neorule can be used accurately and safely to measure newborn infants including sick infants less than 32 weeks $\mathrm{GA}$, and it is recommended that infants be measured using the maximum or mean of three CHL measurements.

\section{ACKNOWLEDGEMENTS}

We would like to dedicate this paper to Neonatal Nurse Consultant Karen Booth who sadly died in December 2001. We thank all mothers in Melbourne and Brighton who kindly allowed us to measure their babies, all other members of staff who helped with the measurements including Drs J Sadowsky and J Carr, and team members in Brighton: Lisa Rooney, Sandra Hobbs, Marie Dudley Ward, Jackie Macdonald, Alison Creasey, Jan Yoxen, Lorna Lindo, Sarah Edwards.

\section{Authors' affiliations}

C J Lawn, R J Chavasse, K A Booth, F J Weir, Trevor Mann Baby Unit, Royal Sussex County Hospital, Brighton, UK

R J Chavasse, M Angeles, Department of Neonatology, Royal Women's Hospital, Carlton, Victoria, Australia

\section{REFERENCES}

1 Babson SG, Bramhall JL. Diet and growth in the premature infant. The effect of different dietary intakes of ash-electrolyte and protein on weight gain and linear growth. J Pediatr 1969;74:890-900.

2 Lucas A. Enteral nutrition. In: Tsang R, Lucas A, Uaay R, et al, eds. Nutritional needs of the preterm infant: scientific basis and practical guidelines. Baltimore: Williams and Wilkins, 1993:209-24.

3 Cooke RWI, Lucas A, Yudkin PLN, et al. Head circumference as an index of brain weight in the fetus and newborn. Early Hum Dev 1977;1:145-9.
4 Sparks JW, Ross JC, Cetin I. Intrauterine growth and nutrition. In: Polin RA, Fox WW, eds. Fetal and neonatal physiology. Philadelphia: WB Saunders, 1992:267-90.

5 Babson SG. Growth of low-birth-weight infants. J Pediatr 1970;77:11-18.

6 Davies DP. Physical growth from fetus to early childhood. In: Davis JA Dobbing J, eds. Scientific foundations of paediatrics. London: William Heinemann, 1981:303-30

7 James DK, Dryburgh EH, Chiswick ML. Foot length: a new and potentially useful measurement in the neonate. Arch Dis Child 1979:54:226-30.

8 Brooke OG, Onubogu O, Heath R, et al. Human milk and preterm formula compared for effects on growth and metabolism. Arch Dis Child 1987;62:917-23.

9 Brooke OG, Alvear J, Arnold M. Energy retention, energy expenditure, and growth in healthy immature infants. Pediatr Res 1979;13:215-20.

10 Gibson AT, Pearse RG, Wales JK. Knemometry and the assessment of growth in premature babies. Arch Dis Child 1993;69(5 Spec No):498-504.

11 Gibson AT, Pearse RG, Wales JKH. Growth retardation after dexamethasone administration: assessment by knemometry. Arch Dis Child 1993;69:505-9

12 Hermanussen M, Seele K. Mini-knemometry: an accurate technique for lower leg length measurements in early childhood. Ann Hum Biol 1997;24:307-13.

13 Michaelson KM, Skov L, Badsberg JH, et al. Short-term measurement of linear growth in preterm infants. Validation of a hand-held knemometer. Pediatr Res 1991; 30:464-8.

14 Griffin IJ, Pang NM, Perring J, et al. Knee-heel length measurement in healthy preterm infants. Arch Dis Child Fetal Neonatal Ed 1999;81:F50-5.

15 Cooke RJ, Perrin F, Moore J, et al. Methodology of nutrient balance studies in the preterm infant. J Pediatr Gastroenterol Nutr 1988;7:434-40.

16 Fomon SJ, Nelson SE. Size and growth. In: Fomon SJ, ed. Nutrition of normal infants. St Louis: Mosby, 1993:36-84.

17 Bland JM, Altman DG. Statistical methods for assessing agreement between two methods of clinical measurement. Lancet 1986:307-11

18 Rosenberg SN, Verzo B, Engstrom JL, et al. Reliability of length measurements for preterm infants. Neonatal Netw 1992;11:23-7.

19 Smith RA, Newcombe RG, Coggins AM, et al. Length measurements in small neonates. Arch Dis Child 1985;60:837-9.

20 Johnson TS, Engstrom JL, Warda JA, et al. Reliability of length measurements in full-term neonates. J Obstet Gynecol Neonatal Nurs 1998;27:270-6.

21 Davies DP, Holding RE. Neonatometer: a new infant length measurer. Arch Dis Child 1972;47:938-20. 